\title{
FUENTES FOSFÓRICAS DE DIFERENTE SOLUBILIDAD PARA FRIJOL COMÚN EVALUADAS POR MÉTODO ISOTÓPICO ${ }^{1}$
}

\author{
Aurelio García Altunaga ${ }^{2}$, Germán Hernández Barrueta ${ }^{2}$, Antonio Nuviola Montoya ${ }^{2}$, Grisel Herrero Echevarría ${ }^{3}$, \\ Nancy Méndez Pérez ${ }^{2}$, Jean Jacques Drevon 4
}

\begin{abstract}
RESUMEN
Fuentes fosfóricas de diferente solubilidad para frijol común evaluadas por método isotópico. Se condujeron experimentos en casa de cristales de la Estación Experimental La Renée ubicada en la provincia Habana en el año 2003. El objetivo fue evaluar fuentes fosfóricas de diferente solubilidad para frijol común (Phaseolus vulgaris L.) utilizando dos suelos, Acrisol y Ferralsol ródico, y la técnica de dilución isotópica con ${ }^{32} \mathrm{P}$. Las fuentes fosfóricas evaluadas fueron roca fosfórica (RF) Trinidad de Guedes, RF parcialmente solubilizada al $50 \%$ con $\mathrm{H}_{2} \mathrm{SO}_{4}$ (producto denominado comercialmente FPA 50) y superfosfato simple (SS) en el Acrisol y superfosfato triple (SFT) en el Ferralsol ródico. Los genotipos de frijol empleados en el Acrisol fueron BAT 477, DOR 364, DOR 390 y Censa, mientras que en el Ferralsol fueron BAT 58, BAT 477, DOR 364 y CC-25-9(N). Se evaluó producción de materia seca y extracción de P y se determinó la fracción (\%) y cantidad de $\mathrm{P}$ en planta derivado de los portadores y $\%$ de eficiencia de uso del fertilizante. La RF en aplicación directa fue poco efectiva mientras que los superfosfatos y el FPA 50 resultaron efectivos en ambos suelos. Hubo diferencia varietal en la respuesta vegetal y en la utilización del P de las fuentes, que también dependió de las características del suelo.
\end{abstract}

Palabras clave: Fósforo, frijol común, isótopo, roca fosfórica, variedades.

\begin{abstract}
Evaluation of phosphatic fertilizers of different solubility for common bean using isotopic methods. Glasshouse experiments were carried out in the Experimental Station La Renée located in Habana province in the year 2003. The objective of this study was to evaluate the effectiveness of phosphorus $(\mathrm{P})$ sources varying in solubility for common bean (Phaseolus vulgaris L.) nutrition using the isotopic dilution method, growing under two soils, Acrisol and Rhodic Ferralsol. The P sources evaluated in both soils were phosphate rock (PR) Trinidad de Guedes and PR partially solubilized at $50 \%$ with $\mathrm{H}_{2} \mathrm{SO}_{4}$, namely FPA 50, whereas single super phosphate (SS) and triple super phosphate (TSP) were used in the Acrisol and Rhodic Ferralsol soils, respectively. The common bean genotypes were BAT 477, DOR 364, DOR 390 and Censa, and BAT 58, BAT 477, DOR 364 and CC-25-9(N) in the Acrisol and Rhodic Ferralsol soils, respectively. Dry matter production, $\mathrm{P}$ extraction, and the fraction and quantity of $\mathrm{P}$ in plants obtained from different $\mathrm{P}$ sources were evaluated, and $\mathrm{P}$ use efficiency of each P fertilizer were calculated. Single and triple super phosphate and FPA 50 were effective $P$ fertilizers in both soils whereas RF was not.
\end{abstract}

Key words: Phosphorus, common bean, isotope, phosphate rock, genotypes.

\section{INTRODUCCIÓN}

El fósforo (P) es un nutriente esencial para las plantas y su deficiencia restringe severamente los rendimientos agrícolas. Los suelos de las regiones tropicales y sub - tropicales predominantemente son ácidos y a menudo muy deficientes en $\mathrm{P}$ y con alta capacidad de adsorción de $\mathrm{P}$, por lo que requieren aportes

1 Recibido: 13 de julio, 2004. Aceptado: 30 de agosto, 2005

2 Estación Experimental La Renée, Instituto de Suelos, Km 33ํㅡㄹ. Carretera Bejucal-Quivicán, Quivicán, La Habana, Cuba. Correo electrónico: larenee@ceniai.inf.cu.

3 Instituto de Ecología y Sistemática, Carretera de Varona Km. 31ํㅡ, Capdevila, Boyeros, AP.8029, C.P. 10800, Ciudad de La Habana, Cuba. Correo electrónico: ecologia.ies@ama.cu

4 Institute National de Recherche en Agronomie (INRA), Montpellier, France. Correo electrónico: drevonjj@ensam.inra.fr 
sustanciales de $\mathrm{P}$ para un óptimo crecimiento de las plantas. Comúnmente, los fertilizantes fosfóricos totalmente solubles son utilizados para corregir las deficiencias de $\mathrm{P}$, pero mucho países en desarrollo deben importarlos y a menudo no están al alcance de pequeños productores. Además, la intensificación de la producción agrícola en estos países necesita la adición de P no sólo para incrementar la producción sino también para elevar la fertilidad fosfórica del suelo y evitar su posterior degradación. Por tanto, es imperativo explorar fuentes alternativas de P (FAO 2004). Así, una fuente alternativa de $\mathrm{P}$ a los fertilizantes fosfóricos totalmente solubles importados es el uso de fosfatos naturales, ya sea aplicados directamente en aquellas condiciones de suelos y cultivos donde resulten eficientes agronómica y económicamente, o modificados, de forma tal que pueda incrementarse la eficiencia del fosfato natural (Kato et al. 1995).

En Cuba, el frijol común (Phaseolus vulgaris L.), principalmente las variedades de color negro, representan un componente esencial de la dieta alimentaria. Sin embargo, el $\mathrm{P}$ es uno de los principales factores que limita sus rendimientos y la eficiencia de la fijación simbiótica del nitrógeno (FSN) en ecosistemas cubanos donde se cultiva esta leguminosa.

Autores como Chien et al. (1993, 1995), Fageria y Carvalho (1996), García et al. (1996) y Gómez et al. (2000) han comprobado el importante papel del P para la nutrición de leguminosas así como la posible utilización de RF como fuente de P. Rocas fosfóricas de diferentes orígenes fueron evaluadas como fuentes de $\mathrm{P}$ para frijol común cultivado en suelos colombianos; las más efectivas, similares al super fosfato triple, resultaron las RF que poseían alta solubilidad en citrato (Hammond 1979).

Sin utilizar la técnica isotópica, Gómez et al. (1996) demostraron la existencia de diferencias genotípicas en frijol común respecto a la eficiencia en el uso del $\mathrm{P}$ y seleccionar aquellos con mayor expresión de este carácter. Por otra parte, como conclusión de un Informe Final de un Proyecto Coordinado de Investigación FAO/IAEA aparece que la utilización de RF es más efectiva en suelos con bajo $\mathrm{pH}$, baja disponibilidad de $\mathrm{P}$, alta capacidad de intercambio catiónico, bajo calcio intercambiable y alto contenido de materia orgánica (Zapata 1999).

Pino et al. (2002) utilizaron la técnica de valor A para determinar la cantidad necesaria de RF Bahía Inglesa para sustituir una unidad de SFT para la nutrición de genotipos de trigo (Triticum aestivum L. y Triticum durum). Afirman que la variabilidad genotípica depende de la fecha de siembra y el suelo. Además, clasificaron los genotipos de trigo de acuerdo a las cantidades de RF que debían emplearse.
Zapata (2000) concluyó lo ventajoso que resulta emplear el isótopo ${ }^{32} \mathrm{P}$ en estudios tales como caracterizar la dinámica del $\mathrm{P}$ en el suelo y en la evaluación de la efectividad agronómica de RF naturales y modificadas.

Beebe et al. (1997) identificaron genotipos de frijol común respecto a su adaptabilidad a deficiencia de $\mathrm{P}$ en el suelo, al ser este uno de los principales factores que limitan la producción de esta leguminosa en suelos tropicales. Entre los resultados más sobresalientes que reportan se encuentran: i) alta variación en la eficiencia de uso del $\mathrm{P}$ entre genotipos para todos los hábitos de crecimiento, ii) identificación de consistente diferencia regional para los genotipos trepadores, y iii) existen evidencias de que la adaptabilidad a bajo $P$ para frijol común se haya adquirido durante o después de su domesticación.

Por otra parte, se ha encontrado evidencia experimental de la existencia de diferencias genotípicas en frijol común en cuanto a producción de granos y fijación del nitrógeno atmosférico al ser cultivados en diferentes suelos. Así, Kipe-Nolt et al. (1999) evaluaron nueve líneas de frijol común en dos suelos, Aquic Hapludoll y Plinthidic Kandiudox, y reportan que los niveles y cantidades de nitrógeno $\left(\mathrm{N}_{2}\right)$ fijado resultaron mayores en el Oxisol. También encontraron que las tres líneas que presentaron mejor comportamiento (BAT 477, RIZ 32 y RIZ 68) en el Oxisol, igualmente resultaron las de mayor fijación de $\mathrm{N}_{2}$ en el suelo Aquic Hapludoll. También Da Silva et al. (1999) encontraron respuesta diferenciada de frijol común cultivado en suelos clasificados como Oxic Paleudalf y Ultisol a la inoculación y fertilización nitrogenada. Las variables de respuesta que consideraron, entre otras, fueron cantidad y peso de los nódulos, y producción de materia seca y de semillas.

Por otra parte, Vadez et al. (1999) encontraron que la variabilidad de fijación de nitrógeno atmosférico en frijol común cultivado bajo deficiencia de $\mathrm{P}$ se encuentra relacionada con la eficiencia en el uso del $\mathrm{P}$, fundamentalmente para los genotipos de tipo IV con floración más tardía, razón por la cual los proponen como progenitores con el propósito de mejorar la FSN en suelos deficientes en $\mathrm{P}$.

La aplicación a voleo de la RF Huila fue más eficiente que su aplicación en bandas para frijol cultivado en Popayán, Colombia. Sin embargo, en el segundo año las diferencias entre tratamientos fueron menores debido a la movilización del P aplicado en bandas por la preparación del terreno antes de la segunda aplicación (Hammond et al. 1986).

La acidulación parcial de la RF Pesca de Colombia, de baja reactividad, a $40 \%$ con $\mathrm{H}_{2} \mathrm{SO}_{4}$ fue un 
método eficiente para obtener rendimientos de frijol de alrededor de $900 \mathrm{~kg} / \mathrm{ha}$, semejante al obtenido al emplear SFT (Chien y Friesen 2000). Por otra parte, Rodríguez (2000) encontró que la aplicación de RF Trinidad de Guedes de Cuba, parcialmente acidulada con $\mathrm{H}_{2} \mathrm{SO}_{4}$ al 50\% (FPA 50), permitió incrementar los rendimientos del genotipo de frijol BAT 304 cultivado en un Ferralsol ródico de reacción neutra.

El objetivo del presente trabajo fue evaluar la eficiencia de portadores de $\mathrm{P}$ de diferente solubilidad como fuentes de $\mathrm{P}$ para genotipos de frijol común, utilizando para ello dos suelos donde se cultiva esta leguminosa y el auxilio de la técnica isotópica.

\section{MATERIALES Y MÉTODOS}

Los suelos utilizados se clasifican como Acrisol y Ferralsol ródico. Algunas de sus características se presentan en el Cuadro 1.

\section{Experimento desarrollado en suelo Acrisol de Pinar del Río}

En el suelo Acrisol de Pinar de Río, las fuentes fosfóricas empleadas fueron: Roca fosfórica (RF) Trinidad de Guedes; RF parcialmente solubilizada al 50\% con $\mathrm{H}_{2} \mathrm{SO}_{4}$ (producto denominado comercialmente FPA 50) y superfosfato simple (SS).

Se evaluaron las variedades de frijol común: BAT 477, DOR 364, DOR 390, Censa.

La capacidad de las macetas fue de $1,4 \mathrm{~kg}$ de suelo y la actividad de ${ }^{32} \mathrm{P}$ aplicada por maceta fue de 2,96 $\mathrm{MBq}+2 \mathrm{mg} \mathrm{P} / \mathrm{kg}$.

\section{Tratamientos}

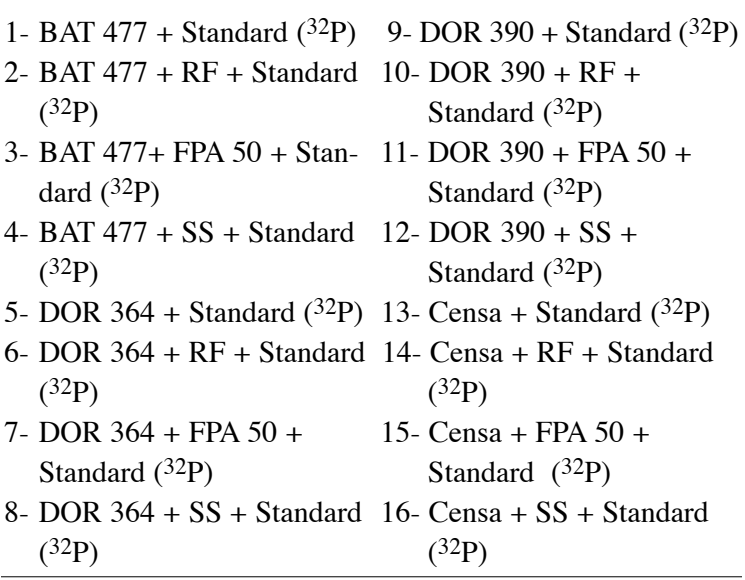

\section{Experimento desarrollado en suelo Ferralsol ródico de La Habana}

En el suelo Ferrasol ródico de la Habana, las fuentes fosfóricas empleadas fueron: Roca fosfórica (RF), Trinidad de Guedes; RF parcialmente solubilizada al $50 \%$ con $\mathrm{H}_{2} \mathrm{SO}_{4}$ (FPA 50) y superfosfato triple (SFT).

Se evaluaron las variedades de frijol común: BAT 58, BAT 477, CC 25-9(N) y DOR 364.

La capacidad de las macetas fue de $1,2 \mathrm{~kg}$ de suelo y la actividad de ${ }^{32} \mathrm{P}$ aplicada por maceta fue de 1,85 $\mathrm{MBq}+5 \mathrm{mg} \mathrm{P} / \mathrm{kg}$.

En los dos experimentos las siguientes especificaciones son comunes:

Semillas por maceta: Se sembraron seis semillas por maceta; pasados cuatro días de la germinación se realizó un raleo y se dejaron tres plantas por maceta.

Cuadro 1. Algunas características de los suelos. La Habana, Cuba. 2003.

\begin{tabular}{|c|c|c|c|c|c|c|c|c|}
\hline & \multirow[t]{2}{*}{ pH KCl } & MO1 & $\mathbf{N}$ total & \multirow{2}{*}{$\begin{array}{c}\text { P disp. } \\
\mathrm{mg} \mathrm{P} / \mathrm{kg}\end{array}$} & $\mathbf{C a}$ & Mg & $\mathbf{K}$ & $\mathbf{N a}$ \\
\hline & & & & & \multicolumn{4}{|c|}{ cmol (+) / kg } \\
\hline Acrisol & 3,80 & 3,02 & 0,115 & 4,4 & 0,75 & 0,43 & 0,05 & 0,10 \\
\hline Ferralsol ródico & 4,70 & 2,82 & 0,119 & 7,2 & 6,77 & 0,36 & 0,57 & 0,10 \\
\hline
\end{tabular}

(1): Materia orgánica, método Walkley-Black; $\mathrm{N}$ total, método Kjeldhal; P disponible, método Bray Kurtz I; Cationes Canjeables, método Schastschabel. 
Cuadro 2. Composición química de la roca fosfórica Trinidad de Guedes (RF) y la RF parcialmente solubilizada (FPA 50) (en \%). La Habana, Cuba. 2003.

\begin{tabular}{lcccccc}
\hline Fuente de $\mathbf{P}^{\mathbf{1}}$ & $\mathbf{P}_{\mathbf{2}} \mathbf{O}_{\mathbf{5}}$ & $\mathbf{C a O}$ & $\mathbf{M g O}$ & $\mathbf{S}$ & $\mathbf{F e}_{\mathbf{2}} \mathbf{O}_{\mathbf{3}}$ & $\mathbf{A l}_{\mathbf{2}} \mathbf{O}_{\mathbf{3}}$ \\
\hline RF & 30,6 & 43,6 & 0,28 & 0,6 & 2,14 & 3,42 \\
FPA 50 & 19,2 & 27,1 & 0,31 & 8,1 & 2,08 & 4,02 \\
\hline
\end{tabular}

(1): FPA 50: RF Trinidad de Guedes parcialmente acidulada con $\mathrm{H}_{2} \mathrm{SO}_{4}$ al 50\%. FPA 50 se produjo en la Empresa Rayonitro, Matanzas, Cuba.

\section{Tratamientos}

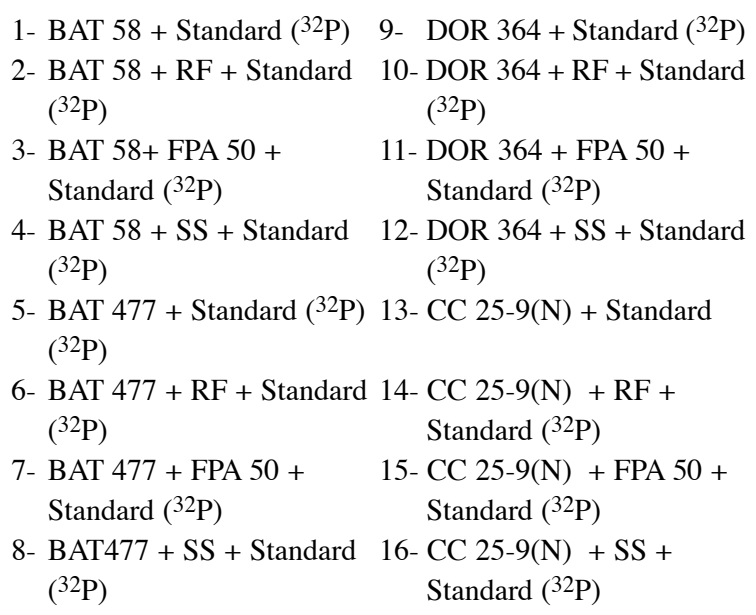

\section{Número de réplicas: tres.}

Las dosis de $\mathrm{P}$ aplicada con cada portador fueron de $100 \mathrm{mg} \mathrm{P} / \mathrm{kg}$ (RF, 13,4 \% P; FPA 50, 8,4 \%P; SS, 8,7 $\%$ P y SFT, $19,6 \% \mathrm{P})$. Las dosis de nitrógeno $(\mathrm{N})$ y potasio $(\mathrm{K})$ aplicadas en cada maceta fueron de $100 \mathrm{mg}$ $\mathrm{N} / \mathrm{kg}$ y $60 \mathrm{mg} \mathrm{K} / \mathrm{kg}$ respectivamente, se utilizó como portadores, la urea $(46 \% \mathrm{~N})$ y el $\mathrm{KCl}\left(60 \% \mathrm{~K}_{2} \mathrm{O}\right)$, respectivamente. El $\mathrm{N}$ y el $\mathrm{K}$ fueron aplicados al germinar las plantas a razón de $20 \mathrm{ml}$ de solución por maceta.

Método isotópico: Valor A. Método indirecto, marcaje del suelo según IAEA (2001).

Durante todo el período vegetativo la humedad de las macetas se mantuvo al $60 \%$ de la capacidad máxima de retención de $\mathrm{H}_{2} \mathrm{O}$ mediante control del peso de las macetas. La parte aérea de las plantas se cosechó pasados 35 días de la siembra, al iniciarse la floración. Las plantas se secaron en estufa a $65^{\circ} \mathrm{C}$ durante 72 horas para determinar la materia seca producida y por digestión por vía seca, la concentración de $\mathrm{P}$ en planta. Una parte del digesto se utilizó para medir la actividad del ${ }^{32} \mathrm{P}$ utilizando equipo de centelleo líquido (efecto Cerenkov).
Determinaciones realizadas: Producción de materia seca (MS, g), concentración de P en planta (\%), actividad de ${ }^{32} \mathrm{P}$ en planta, extracción de $\mathrm{P}$ (por cálculo, $\mathrm{mg} \mathrm{P} / \mathrm{maceta})$.

Las fórmulas utilizadas (IAEA 2001) fueron:

$\%$ Pddf $=(\mathrm{AE}$ del Trat. con Fert. $/$ AE del Trat. sin Fert.) x 100

$\mathrm{AE}($ Actividad específica $)=$ Radiactividad $(\mathrm{dpm}) / \mathrm{mg}{ }^{31} \mathrm{P}$ Valor A suelo $=[(1-\%$ Pddf $) / \%$ Pddf $] \times$ Dosis de $\mathrm{P}$ aplicada

Rendimiento de $\mathrm{P}(\mathrm{Pddf})=(\%$ Pddf $\mathrm{x}$ Extracción de $\mathrm{P}) /$ 100

$\%$ Pddf $=(\%$ Pddf $x$ Extracción de P $) / 100$

$\%$ EUF (\% de Eficiencia de utilización del fertilizante) $=($ Pddf $/ \mathrm{P}$ aplicado $) \times 100$

donde:

\% Pddf: \% de P derivado del fertilizante evaluado

Valor A suelo: Cantidad de P disponible en el suelo expresado en unidades equivalentes del fertilizante utilizado como referencia.

Rendimiento de P: Cantidad de P del fertilizante extraída por la planta.

La Eficiencia Agronómica Relativa (EAR, \%) para la RF y FPA 50 se calculó a partir de los datos de materia seca producida y extracción de $\mathrm{P}$ utilizando la fórmula EAR $(\%)=\left[\left(\mathrm{Y}_{1}-\mathrm{Y}_{0}\right) /\left(\mathrm{Y}_{2}-\mathrm{Y}_{0}\right)\right] \times 100$, donde $\mathrm{Y}_{1}$ = Materia seca o P extraído obtenido con RF o FPA $50, \mathrm{Y}_{2}=$ Materia seca o P extraído obtenido con SS o SFT, Y0 = Materia seca o P extraído obtenido y el tratamiento Control (Chien 2002).

La evaluación estadística de los resultados experimentales comprendió análisis de varianza acorde al diseño experimental empleado, arreglo factorial $4 \times 4$ 
(cuatro tratamientos de $\mathrm{P}$ y cuatro genotipos de frijol común). Las medias de tratamientos se compararon mediante prueba de Newman - Keuls. Se trabajó al 5\% de probabilidad máxima de error. Se utilizó el paquete estadístico STATITCF.

\section{RESULTADOS}

\section{Materia seca}

Los datos en el Cuadro 3 muestran que, independientemente del tipo de suelo, hubo un efecto significativo de los genotipos en el crecimiento. En el Acrisol, la materia seca producida por DOR 390 y Censa (4,90 y 4,77 g mac. ${ }^{-1}$, respectivamente) fue significativamente superior a BAT 477 (3,93 g mac. $\left.^{-1}\right)$. Mientras, en el Ferralsol ródico BAT 58 y CC $25-9 \mathrm{~N}$ (2,88 y 2,80 g mac. $^{-1}$, respectivamente) fueron significativamente superiores a DOR 364 y BAT 477 (2,64 y 2,51 $\mathrm{g} \mathrm{mac}^{-1}$, respectivamente).

Además, en el Ferralsol ródico el efecto de la fuentes de P fue significativo; SFT y FPA 50 (3,00 y 2,97 g mac. $^{-1}$, respectivamente) fueron significativamente superiores que los tratamientos con RF y Control (2,62 y 2,25 g mac. ${ }^{-1}$, respectivamente). La producción de materia seca se incrementó al aumentar el grado de solubilización, pero sin diferencias estadísticas entre FPA y SFT.

\section{Extracción de $\mathbf{P}$}

Se encontró un efecto significativo de la interacción Genotipos x Fuentes de P para la extracción de P en ambos suelos (Cuadro 4). En el Acrisol, la extracción de P en el tratamiento Censa/SS (19,07 $\left.\mathrm{mg} \mathrm{P} \mathrm{mac.-}^{-1}\right)$ fue significativamente mayor que en el resto de las combinaciones Genotipo/Fuente de P; DOR 390 y Censa extraen significativamente más $\mathrm{P}$ cuando se fertilizan con SS (12,82 y 19,07 mg P mac.- ${ }^{-1}$, respectivamente) o FPA 50 (14,83 y $14,48 \mathrm{mg} \mathrm{P} \mathrm{mac.} .^{-1}$, respectivamente) que al fertilizarlos con $\mathrm{RF}\left(9,25\right.$ y $8,54 \mathrm{mg} \mathrm{P}$ mac. $^{-1}$, respectivamente). Es notable que BAT 477, un genotipo desarrollado con adaptabilidad a características de suelos tropicales tales como acidez y baja disponibilidad de $\mathrm{P}$, realice la menor extracción de $\mathrm{P}$ en el tratamiento Control $(6,88 \mathrm{mg} \mathrm{P}$ mac. ${ }^{-1}$ ) que el resto de los genotipos, demostrando de esta forma su alta eficiencia de uso del $\mathrm{P}$ absorbido.

En el Ferralsol ródico el tratamiento DOR 364/SFT $\left(13,56 \mathrm{mg} \mathrm{P} \mathrm{mac}^{-1}\right)$ fue significativamente superior que el resto de la combinaciones Genotipo/Fuente de $\mathrm{P}$.

Cuadro 3. Materia seca $\left(\mathrm{g} \mathrm{mac}^{-1}\right)$ obtenida con varias fuentes de $\mathrm{P}$ para genotipos de frijol común. La Habana, $2003^{1}$.

\begin{tabular}{lccccc}
\hline Acrisol & Control & RF & FPA 50 & SS & Medias de genotipos \\
\hline BAT 477 & 3,82 & 3,50 & 4,02 & 4,40 & $3,93 \mathrm{~b}$ \\
DOR 364 & 4,08 & 4,35 & 4,96 & 4,21 & $4,40 \mathrm{ab}$ \\
DOR 390 & 4,32 & 5,65 & 4,95 & 4,68 & $4,90 \mathrm{a}$ \\
CENSA & 4,39 & 4,62 & 4,98 & 5,11 & $4,77 \mathrm{a}$ \\
Medias de fuentes de P & 4,15 & 4,53 & 4,73 & 4,60 & - \\
Error standard & & & & $0,163^{*}$ & \\
Genotipos & & & $0,163 \mathrm{NS}$ & \\
Fuente de P & & $0,327 \mathrm{NS}$ & \\
Int. Genotipo x Fuente de P. & & &
\end{tabular}

\begin{tabular}{lccccc}
\hline Ferrasol Ródico & Control & RF & FPA 50 & SS & Medias de genotipos \\
\hline BAT 58 & 2,43 & 2,64 & 3,14 & 3,32 & $2,88 \mathrm{a}$ \\
BAT 477 & 2,01 & 2,40 & 3,00 & 2,64 & $2,51 \mathrm{c}$ \\
DOR 364 & 2,13 & 2,81 & 2,77 & 2,86 & $2,64 \mathrm{bc}$ \\
CC 25-9N & 2,41 & 2,64 & 2,97 & 3,17 & $2,80 \mathrm{a}$ \\
Medias de fuentes de P & $2,25 \mathrm{c}$ & $2,62 \mathrm{~b}$ & $2,97 \mathrm{a}$ & $3,00 \mathrm{a}$ & - \\
Error standard & & & & $0,079 *$ & \\
Genotipos & & & $0,091 *$ & \\
Fuente de P & & & $0,129 \mathrm{NS}$ & \\
Int. Genotipo x Fuente de P. & &
\end{tabular}

\footnotetext{
${ }^{1}$ Medias con letras desiguales difieren estadísticamente a $\mathrm{P} \leq 0,05$.

* Prueba de Newman - Keuls.
} 
Cuadro 4. Extracción de $\mathrm{P}\left(\mathrm{mg}\right.$ mac. $\left.{ }^{-1}\right)$ obtenida con varias fuentes de $\mathrm{P}$ para genotipos de frijol común. La Habana, 2003¹.

\begin{tabular}{|c|c|c|c|c|c|}
\hline Acrisol & Control & $\mathbf{R F}$ & FPA 50 & SS & $\begin{array}{l}\text { Medias de } \\
\text { genotipos }\end{array}$ \\
\hline BAT 477 & $6,88 \mathrm{e}$ & 9,77 bcde & 9,80 bcde & $14,07 \mathrm{bc}$ & $10,13 \mathrm{~b}$ \\
\hline DOR 364 & 9,22 cde & 9,23 cde & $12,37 \mathrm{bcd}$ & $12,46 \mathrm{bcd}$ & $10,82 \mathrm{~b}$ \\
\hline DOR 390 & 9,85 bcde & 9,25 cde & $14,83 \mathrm{~b}$ & $12,82 \mathrm{bcd}$ & $11,69 \mathrm{ab}$ \\
\hline CENSA & 9,76 bcde & $8,54 \mathrm{de}$ & $14,48 \mathrm{bc}$ & $19,07 \mathrm{a}$ & $12,96 \mathrm{a}$ \\
\hline Medias de fuentes de P & $8,93 \mathrm{c}$ & $9,20 \mathrm{~b}$ & $12,87 \mathrm{~b}$ & $14,60 \mathrm{a}$ & - \\
\hline \multicolumn{6}{|l|}{ Error standard } \\
\hline Genotipo & \multicolumn{5}{|c|}{$0,548 *$} \\
\hline Fuente de $\mathrm{P}$ & & & \multicolumn{2}{|l|}{$0,548^{*}$} & \\
\hline \multicolumn{3}{|c|}{ Int. Genotipo x Fuente de P } & \multicolumn{2}{|l|}{$1,095^{*}$} & \\
\hline Ferrasol Ródico & Control & $\mathbf{R F}$ & FPA 50 & SS & $\begin{array}{l}\text { Medias de } \\
\text { genotipos }\end{array}$ \\
\hline BAT 58 & $7,32 \mathrm{~d}$ & $8,54 \mathrm{~cd}$ & $9,83 \mathrm{bcd}$ & $9,98 \mathrm{bcd}$ & 8,92 \\
\hline BAT 477 & $7,61 \mathrm{~d}$ & $8,23 \mathrm{~cd}$ & $11,97 \mathrm{ab}$ & $11,12 \mathrm{bc}$ & 9,73 \\
\hline DOR 364 & $7,38 \mathrm{~d}$ & 9,78 bcd & $10,10 \mathrm{bcd}$ & $13,56 \mathrm{a}$ & 10,20 \\
\hline $\mathrm{CC} 25-9 \mathrm{~N}$ & $10,42 \mathrm{bcd}$ & $8,92 \mathrm{bcd}$ & $10,50 \mathrm{bcd}$ & $9,01 \mathrm{bcd}$ & 9,71 \\
\hline \multicolumn{6}{|c|}{ Error standard } \\
\hline Genotipo & \multicolumn{5}{|c|}{$0,359 \mathrm{NS}$} \\
\hline Fuente de $\mathrm{P}$ & \multicolumn{5}{|c|}{$0,414^{*}$} \\
\hline \multicolumn{3}{|c|}{ Int. Genotipo x Fuente de P } & \multicolumn{2}{|l|}{$0,686^{*}$} & \\
\hline
\end{tabular}

${ }^{1}$ Medias con letras desiguales difieren estadísticamente a $\mathrm{P} \leq 0,05$.

* Prueba de Newman - Keuls.

Además, BAT 477 fertilizado con FPA 50 o SFT (11, 97 y $11,12 \mathrm{mg} \mathrm{P}$ mac. $^{-1}$, respectivamente) extraen significativamente más $\mathrm{P}$ que el resto de las combinaciones Genotipo/Fuente de $\mathrm{P}$, excepto el tratamiento DOR 364/SFT (13,56 mg P mac. $\left.{ }^{-1}\right)$. CC 25-9N extrae mayor cantidad de Pen el tratamiento Control que el resto de los genotipos de frijol; este resultado concuerda con García et al. (1996) quiénes reportaron que este genotipo requiere elevadas cantidades de $\mathrm{P}$ para su crecimiento.

\section{Eficiencia Agronómica Relativa}

En el Acrisol la RF fue una fuente eficiente de P para DOR 390 y DOR 364 mientras que FPA 50 lo fue para DOR 364, DOR 390 y Censa puesto que los respectivos valores de Eficiencia Agronómica Relativa (EAR) fueron superiores al valor de 70\% (Cuadro 5).

Los valores de EAR calculados a partir de los datos de extracción de P muestran que FPA 50 fue una fuente eficiente de $\mathrm{P}$ para DOR 390 mientras que la RF no lo fue para ningún genotipo de fríjol (EAR $<70 \%)$. En el Ferralsol ródico, a partir de los datos de materia
Cuadro 5. Eficiencia Agronómica Relativa (EAR, \%) obtenidas con varias fuentes de $\mathrm{P}$ para genotipos de frijol común. La Habana, 2003.

\begin{tabular}{|c|c|c|c|c|}
\hline \multirow[t]{2}{*}{ Acrisol } & \multicolumn{2}{|c|}{$\begin{array}{c}\text { EAR, \% } \\
\text { (calculada a partir } \\
\text { de los datos de } \\
\text { materia seca) }\end{array}$} & \multicolumn{2}{|c|}{$\begin{array}{c}\text { EAR, \% } \\
\text { (calculada a partir } \\
\text { de los datos de } \\
\text { extracción de } \mathbf{P} \text { ) }\end{array}$} \\
\hline & RF & FPA 50 & RF & FPA 50 \\
\hline BAT 477 & 0 & 34 & 40 & 41 \\
\hline DOR 364 & 208 & 677 & 0 & 60 \\
\hline DOR 390 & 369 & 175 & 0 & 168 \\
\hline CENSA & 32 & 82 & 0 & 51 \\
\hline \multirow[t]{2}{*}{ Ferralsol } & \multicolumn{2}{|c|}{$\begin{array}{c}\text { EAR, \% } \\
\text { (calculada a partir } \\
\text { de los datos de } \\
\text { materia seca) }\end{array}$} & \multicolumn{2}{|c|}{$\begin{array}{c}\text { EAR, \% } \\
\text { (calculada a partir } \\
\text { de los datos de } \\
\text { extracción de } \mathbf{P} \text { ) }\end{array}$} \\
\hline & $\mathbf{R F}$ & FPA 50 & RF & FPA 50 \\
\hline BAT 58 & 24 & 80 & 46 & 94 \\
\hline BAT 477 & 62 & 157 & 18 & 124 \\
\hline DOR 364 & 93 & 88 & 39 & 44 \\
\hline CC $25-9 \mathrm{~N}$ & 30 & 74 & 0 & 0 \\
\hline
\end{tabular}

EAR SS $=100 \%$ y EAR SFT $=100 \%$. 
seca, la RF fue una eficiente fuente de P para DOR 364 mientras que FPA 50 resultó eficiente fuente de P para todos los genotipos evaluados. Los valores de EAR calculados a partir de la extracción de $\mathrm{P}$ muestran que la RF no resultó una fuente efectiva de P y que FPA 50 fue efectiva para BAT 477 y BAT 58.

\section{Fracción de $P$ en planta derivada de las fuentes de $P$ (\% Pddf)}

La determinación del parámetro \%Pddf (fracción de $\mathrm{P}$ en planta derivado del fertilizante) para la evaluación de fuentes de $\mathrm{P}$ utilizando el método isotópico tiene la ventaja que el mismo es independiente del rendimiento (Bezzola et al. 1994); el valor límite para considerar una fuente de P como eficiente es $30 \%$ (IAEA 2001). Siguiendo este criterio, en el Acrisol la aplicación de RF fue efectiva para DOR 364 (Pddf > 30\%); FPA 50 y SS fueron efectivas fuentes de $\mathrm{P}$ para todos los genotipos evaluados (Cuadro 6). Por otra parte, los valores de \%Pddf para BAT 477 y DOR 390 correspondientes a los tratamientos con FPA 50 y SS fueron muy similares en cada caso. Estos resultados muestran que ambos genotipos poseen similar mecanismo en la adquisición del P de FPA 50 y SS. En contraste, Censa y DOR 364 muestran mayores valores de \%Pddf cuando fueron fertilizados con SS que con FPA 50. Por tanto, estos resultados demuestran la existencia de diferencia genotípicas en la utilización del $\mathrm{P}$ procedente de fuentes de diferente solubilidad. En el Ferralsol ródico la RF no participó significativamente en la nutrición fosfórica de los genotipos evaluados puesto que los valores de \%Pddf resultaron menores que $30 \%$. Contrariamente, FPA y SFT fueron efectivas fuentes de P para todos los genotipos (Cuadro 6).

Cuadro 6. Fracción de $\mathrm{P}$ en planta derivada de la fuente de $\mathrm{P}$ (\%Pddf) obtenidas con varias fuentes de $\mathrm{P}$ para genotipos de frijol común. La Habana, 2003.

\begin{tabular}{lccc}
\hline \multicolumn{1}{c}{ ACRISOL } & RF & FPA 50 & SS \\
\hline BAT 477 & 29 & 40 & 39 \\
DOR 364 & 32 & 47 & 56 \\
DOR 390 & 16 & 34 & 37 \\
CENSA & 16 & 34 & 60 \\
& & & \\
\hline FERRALSOL & RF & FPA 50 & SFT \\
\hline & & & \\
BAT 58 & 8 & 33 & 40 \\
BAT 477 & 19 & 59 & 70 \\
DOR 364 & 10 & 39 & 56 \\
CC 25-9N & 21 & 32 & 41 \\
\hline
\end{tabular}

\section{Eficiencia de Uso del Fósforo (EUF)}

En el Acrisol, para todos los genotipos de frijol, la RF presentó los menores valores de EUF, de 0,9 a 2,3 $\%$, que FPA 50 , de 2,8 a $4,1 \%$ y SS, de 3,0 a $8,2 \%$ (Cuadro 7). Se encontraron diferencias genotípicas respecto al parámetro EUF: FPA 50 fue una fuente más efectiva para DOR 364 (4,1\%) y DOR 390 (4,0\%), ambos genotipos desarrollados con resistencia al Virus del Mosaico Dorado, que el SS (3,0 y 3,4 \%, respectivamente). Contrariamente, SS fue una fuente más efectiva para BAT $477(4,0 \%)$ y Censa $(8,2 \%)$ que FPA 50 ( 2,8 y $3,5 \%$, respectivamente). El parámetro EUF reveló también la variabilidad en la efectividad de las fuentes evaluadas: los valores de EUF cubrieron el rango de $0,9 \%$ a $2,3 \%$ para la RF, de $2,8 \%$ a $4,1 \%$ para FPA 50 y de $3,0 \%$ a $8,2 \%$ para SS. En el Ferralsol los valores de EUF se incrementaron con el aumento del grado de solubilización de las fuentes de P: EUF - SFT > EUF FPA $50>$ EUF - RF (Figura 1B). Para los dos BAT se encontró intra e inter diferencias genotípicas en los valores de EUF: para BAT 477 los valores de EUF para RF, FPA 50 y SFT fueron dos veces mayores que los debidos a BAT 58, indicando probablemente dos mecanismos diferentes de adquisición del $\mathrm{P}$.

Cuadro 7. Eficiencia de Uso del Fertilizante (EUF, \%) obtenidas con varias fuentes de $\mathrm{P}$ para genotipos de fríjol común. La Habana, 2003.

\begin{tabular}{|c|c|c|c|}
\hline ACRISOL & $\mathbf{R F}$ & FPA 50 & SS \\
\hline BAT 477 & 1,3 & 2,8 & 4,0 \\
\hline DOR 364 & 2,3 & 4,1 & 3,0 \\
\hline DOR 390 & 0,9 & 4,0 & 3,4 \\
\hline CENSA & 1,0 & 3,5 & 8,2 \\
\hline FERRALSOL & $\mathbf{R F}$ & FPA 50 & SFT \\
\hline BAT 58 & 0,6 & 2,7 & 3,3 \\
\hline BAT 477 & 1,3 & 5,9 & 6,5 \\
\hline DOR 364 & 0,7 & 3,4 & 4,2 \\
\hline CC $25-9 \mathrm{~N}$ & 1,6 & 2,8 & 3,1 \\
\hline
\end{tabular}

\section{Tasas de sustitución para RF y FPA 50}

Independientemente del suelo, los valores de las Tasas de Sustitución (TS) para la RF fueron superiores a los de FPA 50 para todos los genotipos de fríjol común evaluados (Cuadro 8). En el Acrisol se encontraron diferencias genotípicas respecto a los valores de TS de la RF 
Cuadro 8. Tasas de Sustitución (TS) o kg de P aplicados como RF o FPA 50 equivalentes a $1 \mathrm{~kg}$ de $\mathrm{P}$ aplicado como super fosfato simple (Acrisol) o Super fosfato triple (Ferralsol) obtenidas para genotipos de frijol común. La Habana, 2003.

\begin{tabular}{lcc}
\hline ACRISOL & RF & FPA 50 \\
\hline BAT 477 & 1,6 & 1,0 \\
DOR 364 & 2,7 & 1,4 \\
DOR 390 & 3,0 & 1,1 \\
CENSA & 7,8 & 2,9 \\
& & \\
\hline FERRALSOL & RF & FPA 50 \\
\hline & & \\
BAT 58 & 7,7 & 1,4 \\
BAT 477 & 9,9 & 1,6 \\
DOR 364 & 11,4 & 2,0 \\
CC 25-9N & 2,6 & 1,5 \\
\hline
\end{tabular}

y FPA 50; para BAT 477, DOR 364 y DOR 390 los valores de TS fueron 1,6; 2,7 y 3,0 (kg de P aplicados como RF equivalentes a $1 \mathrm{~kg}$ de $\mathrm{P}$ aplicado como SS) mientras que para Censa el valor de TS fue 7,8. Los valores de TS para BAT 477, DOR 390 y DOR 364 fueron bastantes cercanos a la unidad, indicando que para estos genotipos la RF fue tan efectiva como el SS. En el Ferralsol el valor TS de la RF para CC $25-9 \mathrm{~N}$ fue más bajo $(2,6)$ que los correspondientes a BAT $58(7,7)$, BAT $477(9,9)$ y DOR $364(11,4)$. Para todos los genotipos de frijol común FPA 50 fue tan efectivo como el SFT porque los valores de TS cubrieron el rango de 1,4 (BAT 58) a 2,0 (DOR 364) (Cuadro 8). Estos resultados revisten importancia al considerar que FPA 50 es un fertilizante fosfórico producido localmente y es más barato que los fertilizantes fosfóricos importados.

\section{DISCUSIÓN}

Se revelaron diferencias en crecimiento entre los genotipos de frijol común evaluados debidas a fuentes de $\mathrm{P}$, genotipos y tipo de suelo.

Respecto a las fuentes de $\mathrm{P}$, en términos globales la RF tuvo poca efectividad de acuerdo a los resultados obtenidos para todos los parámetros de planta evaluados. Por otra parte, la aplicación de RF parcialmente solubilizada (FPA 50) fue más efectiva que la de RF en aplicación directa; una ventaja de FPA 50 sobre la RF es su elevado contenido de S (Cuadro 2). Muchos suelos han recibido durante años aportes de SFT y en la actualidad presentan deficiencias de S. Este resultado es interesante puesto que FPA 50 se evalúa con el objetivo de valorar si puede resultar un sustituto de uso de SFT, este último fertilizante importado y más costoso. Existió correspondencia con otros reportes sobre la casi generalizada poca efectividad de la RF en aplicación directa y en el incremento de la misma cuando la RF es modificada mediante solubilización parcial. Investigaciones desarrolladas en el IFDC (anteriormente International Fertilizer Development Center; actualmente International Center for Soil fertility and Agricultural Development) para determinar si RF de diferentes orígenes podían ser utilizadas para preparar RF parcialmente solubilizadas con efectividad agronómica, concluyeron que, bajo ciertas condiciones, la solubilización parcial es suficiente para alcanzar tal objetivo y que los productos obtenidos son comparables a los fertilizantes fosfóricos totalmente solubles (Chien y Menon 1995 a, b). No obstante, prestan atención a los contenidos de óxidos de hierro y aluminio del material de partida al considerar que los mismos pueden inmovilizar una fracción del fosfato disuelto.

Se encontraron diferencias en la utilización del $\mathrm{P}$ proveniente de fuentes de diferente solubilidad entre los genotipos de frijol común, incluyendo aquellos desarrollados para suelos tropicales (adaptabilidad a acidez del suelo, deficiencia de P, toxicidad de aluminio...) como BAT 58 y BAT 477 y DOR 364 y DOR 390 desarrollados con resistencia a Virus del Mosaico Dorado; además, estas diferencias fueron dependientes también de las características del suelo.

Diversos autores (Chien et al. 1993; Fageria y Carvalho 1996; Chien y Menon 1995 a) demostraron la posibilidad de utilizar RF para la nutrición de leguminosas y la importancia del $\mathrm{P}$ para este cultivo. Por medio de la técnica isotópica (Mahisarakul y Pakkong 2002) evaluaron la habilidad de RF de diferentes orígenes como fuentes de $\mathrm{P}$ para una leguminosa, soya (Glycine max L.); sin embargo, contrariamente a los resultados encontrados con fríjol común y citados anteriormente, la soya no respondió a la aplicación de RF. Estos resultados confirman la alta sensibilidad de frijol común a la nutrición fosfórica y, al mismo tiempo, que la soya es menos sensitivo al $\mathrm{P}$ que el frijol común.

El criterio de \%Pddf, por ser una variable independiente de la respuesta vegetal obtenida, debe predominar sobre \%EUF, el cual depende de la respuesta vegetal obtenida.

La utilidad de emplear la técnica isotópica para establecer diferencias genotípicas y evaluar la participación de una RF reactiva en la nutrición fosfórica de genotipos de caupí (Vigna sp.) ha sido reportado por 
(Ankomah et al. 1995). Estos autores afirman que esta técnica permite realizar comparaciones en el comportamiento de los genotipos evaluados y determinar la cantidad equivalente de esta fuente que muestra similar efectividad que una unidad de super fosfato.

En conclusión, este trabajo se desarrolló para estudiar la efectividad de la roca fosfórica (RF) Trinidad de Guedes, natural y parcialmente solubilizada (FPA 50) como fuentes de $\mathrm{P}$ para genotipos de fríjol común en comparación a fertilizantes fosfóricos totalmente solubles utilizando dos suelos. Cada fuente de P evaluada mostró un comportamiento diferenciado de acuerdo a tres factores, i) características de la fuente de $\mathrm{P}$, ii) genotipo de fríjol común y iii) características del suelo. La poca efectividad de la RF está asociada con su poca solubilidad y elevados contenidos de óxidos de hierro y aluminio. FPA 50 fue una efectiva fuente de P para todos los genotipos de fríjol evaluados en ambos suelos; la solubilización parcial de la RF incrementó su efectividad debido al aumento de la solubilidad del material de partida, la RF. En el Acrisol, entre 1,0 y 2,9 kg de P como FPA 50 equivalen a $1 \mathrm{~kg}$ de $\mathrm{P}$ como SS. En el Ferralsol, entre 1,4 y 2,0 kg de P como FPA 50 equivalen a $1 \mathrm{~kg}$ de $\mathrm{P}$ como SFT. Las diferencias en la respuesta de las plantas a las fuentes de P de genotipos desarrollados con similares caracteres (por ejemplo, BAT 58 y BAT 477 y DOR 364 y DOR 390) puede deberse a un decrecimiento o a la pérdida del carácter. En el Acrisol la mejor combinación fue Censa/SS; BAT 477 posee una alta eficiencia de uso del P. En el Ferralsol la mejor combinación fue DOR 364/SFT; CC 25-9N presenta un alto requerimiento de $P$.

Estos resultados deben ser validados en experimentos en campo. No obstante, los resultados obtenidos resultan útiles para programas de mejoramientos en los que se pretenda obtener genotipos de fríjol común con alta eficiencia de uso del $\mathrm{P}$ proveniente de fuentes de escasa (RF) o mediana (FPA 50) solubilidad o para ser cultivados en suelos deficientes en $P$.

\section{AGRADECIMIENTOS}

Al Organismo Internacional de Energía Atómica (OIEA) por el apoyo técnico y financiero aportado mediante el Contrato de Investigación CUB/10956 perteneciente al CRP "Desarrollo de prácticas de manejo para una producción sostenible en sistemas de cultivos en suelos ácidos tropicales mediante el uso de técnicas nucleares y conexas" (D1.50.06).

\section{LITERATURA CITADA}

ANKOMAH, A.B.; ZAPATA, F.; DANSO, S.K.; AXMANN, H. 1995. Cowpea varietal differences in uptake of phosphorus from Gafsa phosphate rock in a low - P Ultisol. Fertilizer Research 41: 219 - 225.

BEEBE, S.; LYNCH, J.; GALWEY, N.; TOHME, J.; OCHOA, I. 1997. A geographical approach to identify phosphorusefficient genotypes among landraces and wild ancestors of common bean. Euphytica 95: 325 - 336 .

BEZZOLA, L. C.; LÓPEZ, S. C.; BÁRBARO, N,O. 1994. Effectiveness of different phosphatic fertilizers measured using labelled super phosphate and phosphorus taken up by plants. Fertilizer Research 39:31-37.

CHIEN, S.H.; CARMONA, G.; MENON, R. G.; HELLUMS, D.T. 1993. Effect of phosphate rock sources on biological nitrogen fixation by soybean. Fertilizer Research $34: 153-159$.

CHIEN, S. H.; MENON, R.G.; TAYLOR, R.W.; SISTANI, K.R. 1995. Evaluation of agronomic effectiveness of phosphates rocks for aluminium-tolerant soybean cultivar. Communications in Soil Science and Plant Analysis 26 (19-20): 121-131.

CHIEN, S. H.; MENON, R. G. 1995 a. Factors affecting the agronomic effectiveness of phosphate rock for direct application. Fertilizer Research 41: 227 - 234.

CHIEN, S. H.; MENON, R. G. 1995 b. Agronomic evaluation of modified phosphate rock products: IFDC's experience. Fertilizer Research 41: 197 - 209.

CHIEN S, H.; FRIESEN, D. K. 2000. Phosphate fertilisers and management for sustainable crop production in tropical acid soils. In: IAEA - TECDOC - 1159. Management and conservation of tropical acid soils for sustainable crop production. Proceedings of a consultants meeting organized by the Joint FAO/IAEA Division of Nuclear Technique in Food and Agriculture and held in Vienna. p. 73 - 89.

CHIEN, S. H. 2002. Evaluation of available phosphorus and cadmium associated with phosphate rock for direct application. Assessment of soil phosphorus status and management of phosphatic fertilisers to optimize crop production. Vienna, Austria. IAEA - TECDOC-1272: 54 - 72.

DA SILVA, P. M.; TSAI, S.M.; BONETTI, R. 1999. Respuesta a la inoculación y a la fertilización nitrogenada para incrementar la producción y la fijación biológica de nitrógeno en frijol común (Phaseolus vulagris L.). In: J.J. Peña-Cabriales; F. Zapata eds. Aumento de la fijación biológica del nitrógeno en el frijol común en América Latina. ARCAL. México. p. 137 - 144. 
FAGERIA, N. K; de CARVALHO, A. M. 1996. Response of common bean to phosphorus on acid soils. Communications in Soil Science and Plant Analysis 27(5-8): 1447-1458.

FAO. 2004. Use of phosphate rocks for sustainable agriculture. FAO Fertilizer and Plant Nutrition Bulletin 13. Food and Agriculture Organization of the United Nations, Rome, Italy. 2004. 148 p.

GARCÍA, A.; HERNÁNDEZ, G.; NUVIOLA, A.; TOSCANO, V. 1996. Efecto del P sobre el rendimiento y extracción del NP de frijol cultivado en tres suelos. Agronomía Mesoamericana 7(1): 99 - 102.

GÓMEZ, L.; VADEZ, V.; HERNÁNDEZ, G.; SÁNCHEZ, T.; TOSCANO, V. 1996. Eficiencia del uso del fósforo en diez genotipos de frijol común (Phaseolus vulgaris L.). Agronomía Mesoamericana 7(1): 41 - 46.

GÓMEZ, L.; DUEÑAS, G.; SÁNCHEZ, T. 2000. Fijación de $\mathrm{N}_{2}$ en caupí y fríjol común sometidos a estrés de fósforo (P). In: Juan José Peña - Cabriales ed. La fijación biológica de nitrógeno en América Latina: el aporte de las técnicas isotópicas. ARCAL. Irapuato, México. p. $77-88$.

HAMMOND, L. L. 1979. Agronomic measurement of phosphate rock effectiveness, Seminar on Phosphate Rock for Direct Application. Special publication IFDC - S1. International fertilizer Development Center (IFDC), Muscle Shoals, Alabama, EE. UU. p. 147 - 173.

HAMMOND, L. L.; CHIEN, SH; MOKWUNYE, AU. 1986. Agronomic value of unacidulated and partially acidulated phosphate rocks indigenous to the tropics. Advances in Agronomy 40: 89 - 140.

IAEA (International Atomic Energy Agency). 2001. Use of isotopes and radiation methods in soil and water management and crop nutrition. Manual. Training Course Series 14. International Atomic Energy Agency, Vienna, Austria, 247 p.

KATO, N.; ZAPATA, F.; AXMANN, H. 1995. Evaluation of the agronomic effectiveness of natural and partially acidulated phosphate rocks in several soils using ${ }^{32} \mathrm{P}$ isotopic dilution techniques. Fertilizer Research 41:235 - 242.
KIPE-NOLT, J. A.; VARGAS, H.; GILLER K. 1999. Fijación de nitrógeno en líneas mejoradas de Phaseolus vulgaris L. In: J.J. Peña-Cabriales; F. Zapata eds. Aumento de la fijación biológica del nitrógeno en el frijol común en América Latina. ARCAL. Irapuato, México. p. 113 117.

MAHISARAKUL, J.; PAKKONG, $\mathrm{P}$. The use of ${ }^{32} \mathrm{P}$ radioisotopes techniques for evaluating the relative agronomic effectiveness of phosphate rock materials in a soybean - maize crop rotation in acid soils of Thailand. In: IAEA - TECDOC - 1272. Assessment of soil phosphorus status and management of phosphatic fertilizers to optimise crop production. International Atomic Energy Agency, Vienna, Austria. 237 - 249. 2002.

PINO, I.; PARADA, A. M.; ZAPATA, F.; NAVIA, M.; LUZIO, W. 2002. Comparative study on P uptake and utilization from $\mathrm{P}$ fertilizers by Chilean wheat genotypes in volcanic ash soils. In: Assessment of soil phosphorus status and management of phosphatic fertilizers to optimize crop production. IAEA-TECDOC-1272: 156 163.

RODRÍGUEZ, R. 2000. Fertilización de frijol común con roca parcialmente acidulada en suelos ferralíticos rojos compactados de $\mathrm{pH}$ neutral. Tesis presentada en opción al grado científico de doctor en Ciencias. San José, La Habana. Instituto Nacional de Ciencias Agrícolas. 98 p.

VADEZ, V.; LASSO, J. H.; BECK, D. P.; DREVON, J. J. 1999. Variability of $\mathrm{N}_{2}$ - fixation in common bean (Phaseolus vulgaris L.) under P deficiency is related to P use efficiency. Euphytica 106: $231-242$.

ZAPATA, F. 1999. The use of nuclear and related techniques for evaluating the agronomic effectiveness of phosphate fertilizers, in particular rock phosphates. Final Report of the FAO/IAEA Co-ordinated Research Project. FAO/IAEA Division of Nuclear Techniques in Food and Agriculture, Vienna, Austria.

ZAPATA, F. 2000. Evaluating agronomic effectiveness of phosphate rocks using nuclear and related techniques: results from an FAO/IAEA Coordinated Research Project. In: Management and conservation of tropical acid soils for sustainable crop production. IAEA-TECDOC1159: $91-100$. 